\title{
Alginate encapsulation induce colony formation with umbilical cord-derived mesenchymal stem cells
}

\author{
Erkan Gumus ${ }^{1}$. Burcin Irem Abas ${ }^{2}$, Evrim Cevik$^{3}, \quad$ Bilge Kocabiyik $^{4}$, Melike Cenik ${ }^{4}$, \\ Ozge Cevik ${ }^{2}$ \\ ${ }^{1}$ Department of Histology and Embryology, Aydin Adnan Menderes University, School of Medicine, Aydin, Turkey \\ ${ }^{2}$ Department of Biochemistry, Aydin Adnan Menderes University, School of Medicine, Aydin, Turkey \\ ${ }^{3}$ Department of Machinery and Metal Technologies, Aydin Adnan Menderes University, Kocarli Vocational High School, \\ Aydin, Turkey \\ ${ }^{4}$ Department of Molecular Biotechnology, Aydin Adnan Menderes University, Graduate School of Health Sciences, Aydin, \\ Turkey
}

\section{ABSTRACT}

Aim: The umbilical cord (UC) is a rich source of mesenchymal stem cell (MSC) isolation. Since the MSCs isolated from here have high self-renewal capacity and differentiation potential, production through biofabrication is essential for clinical treatments. For the cells to be stored for a long time and presented ready for use, encapsulation is required. In this study, UC-MSC cells were encapsulated with alginate using three different methods: alginate drop, alginate coating, and alginate sphere.

Methods: The cell viability, live/dead cell ratio, and colony formation capacities of the encapsulated cells were examined for 14 days.

Results: In the study, it was found that the most effective method was the alginate sphere form and that the structure of the cells should be preserved by injecting them into biomaterials in encapsulation. Colony formation potential was found to be high in biomaterials with alginate spheres.

Conclusion: As a result, the preservation of UC-MSC cells with alginate sphere encapsulation via biofabrication and their clinical use availability may be beneficial for treating of many diseases.

Keywords: Alginate, hydrogel, mesenchymal stem cell, umbilical cord.

$\triangle$ Dr. Ozge Cevik

Department of Biochemistry, Aydin Adnan Menderes

University, School of Medicine, Aydin, Turkey

E-mail: ozge.cevik@adu.edu.tr

Received: 2021-01-20 Revised: 2021-01-30

Accepted: 2021-02-08 / Published online: 2021-04-01

\section{Introduction}

Mesenchymal stem cells (MSCs) are multipotent cell types obtained from a wide variety of tissues such as adipose tissue, bone marrow, dental pulp, placenta, and umbilical cord [1]. MSCs are different from other cells with its features of proliferation, differentiation, and self-renewal. Although it plays a vital role in development during the embryonic period, they now have the therapeutic potential [2-4]. MSCs have been challenging a role therapeutics in clinical ameliorating from cancer to central nervous system diseases $[5,6]$. MSCs can inhibit the immune system, increase cell proliferation, induce angiogenesis, or migrate toward damaged tissues. Furthermore, the multifunctional capabilities of MSCs increase their use in both the medical and pharmaceutical industries. In the world, stem cell and cellular therapy companies make 
significant investments in obtaining MSCs and keeping them for a long time without disturbing their activity [7]. The umbilical cord (UC) is a non-invasive and ethically trouble-free source of stem cells, as it is isolated from tissue taken at birth. UC contains MSC cells with high availability and high growth capacity of embryonic origin. Compared to cells taken from adult tissues, UC cord cells are a very attractive resource as they have spread faster $[8,9]$.

Isolation processes of MSCs from UC are laborious and higher costly, require a great deal of experience. Isolations can be made differently; the most used methods are enzymatic digestion for separation or explant culture method [10]. After isolation of MSCs, studies are carried out to be stored for a long time and used therapeutically. Biopolymers with high biocompatibility such as alginate, chitosan, agarose, collagen, poly(lactic-coglycolic acid) (PLGA), poly(ethylene glycol) (PEG), (poly(lactic acid) (PLA) and poly(glycolic acid) (PGA) are used for microencapsulation of MSCs [11]. Alginate is a natural polysaccharide and a biopolymer with high biocompatibility, stability, and nonantigenicity. Alginate shows gelation by crosslinking with calcium ions. These formed hydrogels are used as encapsulation material and can be produced by bio fabrication in various applications. Their use in medicine and pharmacy has been increasing rapidly in recent years due to its non-toxicity and gelling. Microencapsulation of UC-derived MSCs with a highly biocompatible polymer such as alginate after isolation may increase their use in stem cell treatments. New microencapsulation methods should be developed in order for UCderived MSCs to maintain their rich content and rapid growth capacity without losing their capabilities. In this study, we investigated the colony formation potential of UC-MSCs in the encapsulation of alginate

\section{Materials and Methods}

\section{Cell culture and conditions}

Human umbilical cord-derived mesenchymal stem cells (UC-MSC) were provided by ATCC (PCS-500-010). Cells were cultured in DMEM (Dulbecco's Modified Eagle's medium) (Gibco, USA) supplemented with $15 \%$ fetal bovine serum (FBS; Gibco), 2 mM L-glutamine, and $100 \mathrm{U} / \mathrm{mL}$ penicillin, and $100 \mu \mathrm{g} / \mathrm{mL}$ streptomycin, at $37^{\circ} \mathrm{C}$ in a humidified atmosphere containing 5\% CO2. The medium was changed every three days.

\section{Preparation of alginate constructs}

Sodium alginate was purchased from Sigma (W201502) and dissolved with sterile PBS (phosphate-buffered saline) solution. Alginate solution was modified prepared different concentration as $0.625 \%, 1.25 \%$ and $2.5 \% \mathrm{w} / \mathrm{v}$ via encapsulation methods [12]. Each solution was passed through 0.45 sterile filters. Sterile calcium chloride solutions $(2 \% \mathrm{w} / \mathrm{v})$ were prepared and used for reducing agent to crosslink the alginate at $25{ }^{\circ} \mathrm{C}$. Calcium and alginate complex was left for 5 minutes to form a hydrogel, and then it was taken into DMEM medium. The alginate encapsulation's rheological behaviors were determined using a rheometer (HAAKE MARS 40 Rheometer, Invitrogen). The viscosity measurements were done at $25^{\circ} \mathrm{C}$ and calculated by shear rate. Alginate deformation rate was calculated as the change in viscosity over 14 days without cells at $37^{\circ} \mathrm{C}$.

\section{Encapsulation of $\boldsymbol{U C}$-MSC}

Experimental design on encapsulation was included in Figure 1. The encapsulation method 
was modified by the amount of alginate and calcium and according to the selected cell [13]. Alginate Drop: Alginate solution (1.25\%) and UC-MSC cells (500 cells) were mixed. $10 \mu \mathrm{L}$ volume of the mixture was dropped into calcium solution. It was incubated for 5 minutes to crosslink, and after washing with PBS, 100 $\mu \mathrm{L}$ of cell medium was added into each well of the 96-well plate. Alginate Coating: $50 \mu \mathrm{L}$ alginate solution $(1.25 \%)$ was coated into each well of the 96-well plate, and $50 \mu \mathrm{L}$ calcium solution was added to crosslink. After the washing with PBS, UC-MSC cells (500 cells) were seeded with $100 \mu \mathrm{L}$ of cell medium was added into each well of the 96-well plate. Alginate Sphere: $10 \mu \mathrm{L}$ alginate solution $(1.25 \%)$ was added into each well of the 96well plate, and $25 \mu \mathrm{L}$ calcium solution was added to the alginate. After the crosslinking, UC-MSC cells ( 500 cells) were injected into the alginate sphere, and $100 \mu \mathrm{L}$ of cell medium was added into each well of the 96-well plate.

\section{Cell viability and live/dead cells}

The encapsulated UC-MSC cells were incubated for 7 days and 14 days. At the end of the incubation, alginate encapsulations were dissolved in sodium citrate-EDTA buffer (55 mM Na-citrate, $20 \mathrm{mM}$ EDTA). Each well was incubated with $10 \mu \mathrm{L}$ MTT solution (MTT; Vybrant, Invitrogen) for 4 hours at $37^{\circ} \mathrm{C}, 5 \%$ $\mathrm{CO} 2$. After incubation, solubilization was done in $100 \mu \mathrm{L}$ SDS buffer to formazan precipitates [14]. Color changes were measured at $570 \mathrm{~nm}$ using a microplate reader (Epoch, Biotek). Dissolved cells were suspended in PBS and measured in a cell counter (Invitrogen Countess II).

\section{Colony formation}

The encapsulated cells were examined morphologically [14] at the end of the 14th day under an inverted microscope (Zeiss Axiovert, Germany). The areas inside the capsules in the cells were focused. The ratio of colony-forming cells among total cells was calculated in each well.

\section{Statistical analyses}

Data were analyzed with GraphPad Prism software (GraphPad Inc., San Diego, CA, USA). All values were presented as mean $\pm \mathrm{SD}$. Between study groups, the obtained data were compared by using a non-paired t-test and twoway ANOVA. Differences were considered statistically significant if the p-value was less than 0.05 .

\section{Results}

Alginate viscosity was measured in a shear rate range of $1-1000 \mathrm{~s}^{-1}$ by increasing the shear rate every $10 \mathrm{~s}$ for $1 \mathrm{~min}$. For $2.5 \%$ alginate, the low shear viscosity at $25{ }^{\circ} \mathrm{C}$ was found to be 282 $\mathrm{mPa} \mathrm{s}$; for $1.25 \%$ alginate, the moderate shear viscosity at $25{ }^{\circ} \mathrm{C}$ was $365 \mathrm{mPa}$; for $0.625 \%$ alginate, the high shear viscosity at $25{ }^{\circ} \mathrm{C}$ was $687 \mathrm{mPa}$ s (Fig 2a). The effect of shear stress on the viscosity was different for each alginate. In alginate deformation, the deformation rate was significantly higher in the coating of $0.625 \%$ alginate on the well surface $(p<0.0001)$ while there is no deformation in the other concentration for encapsulation with drop and sphere of alginate (Fig 2b). Cell viability activities of UC-MSC cells were checked on the 7 th day, as the adhesion and attachment of stem cells were difficult. A certain number of stem cells were planted in each well, it was observed that the cells maintained their viability in the alginate drop group $(p>0.1350)$, but cell viability decreased significantly in the alginate coating group compared to the control $(p<0.0001)$. The alginate sphere group 


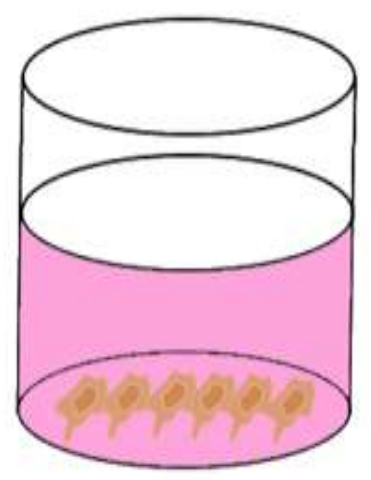

Control UC-MSC
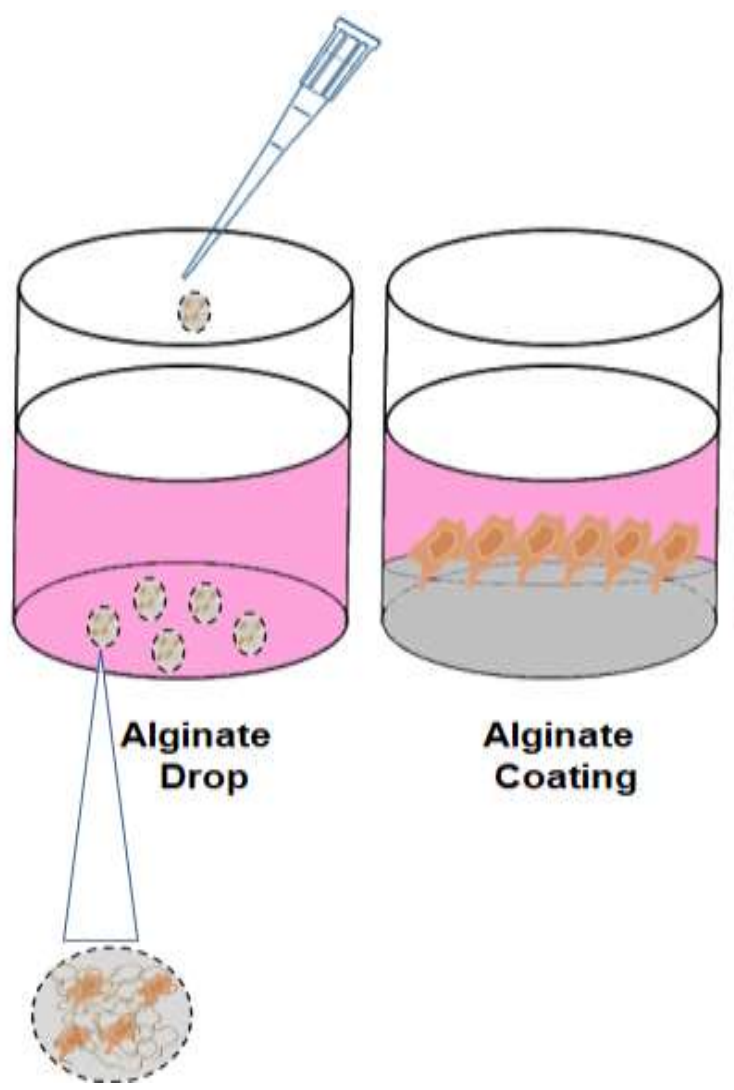

Alginate

Coating

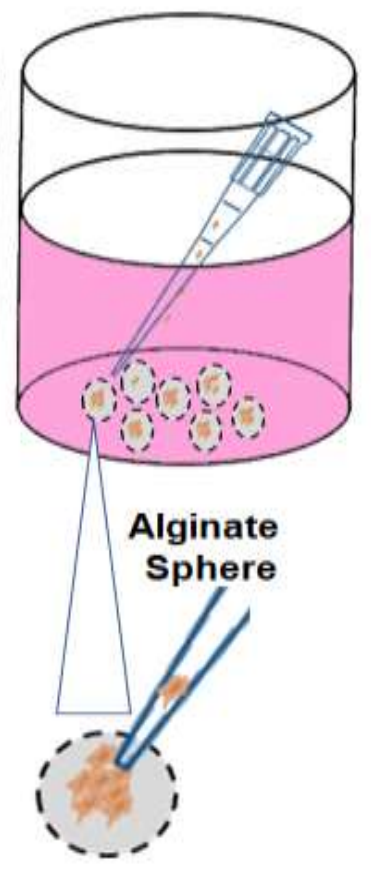

Figure 1. Experimental design for the umbilical cord- mesenchymal stem cells (UC-MSCs).

observed that the cell viability levels increased significantly compared to the control group ( $p<0.0098$, Fig 3a). The live and dead cell proportions of the UC-MSC cells after 14 days were evaluated. It was observed that the number of dead cells increased slightly in the alginate drop group compared to the control group ( $p<0.0166$ ), and the change in the number of live cells was not significant $(p>0.0594)$. It was observed that the number of dead cells increased significantly in the alginate coating group $(p<0.0001)$ and decreased in the alginate coating group of living cells compared to the control group $(p<0.0001)$. It was observed that the number of live cells increased significantly in the alginate sphere group compared to the control group $(p<0.0403)$, and there was no change in the number of dead cells $(p>0.1708$, Fig 3b).

The colony formation potential of UC-MSC cells after 14 days was evaluated microscopically (Fig 4). Since cells in the control group tend to adhere to cell culture plates, their colony-forming potential is limited. The colony-forming potential of cells in the alginate drop increased significantly compared to control group cells $(p<0.0002)$. When the alginate coating group was compared with the control group, it was observed that there was no change in colony formation potential $(p>0.3780)$. The colony-forming potential of UC-MSC cells in the alginate sphere increased significantly compared to control group cells ( $p<0.0001$, Fig 4b).

\section{Discussion}

MSCs are rich in content, and they interact with other cells through bioactive mediators in their structure, such as hormones, growth factors, cytokines, and extracellular vesicles that exert angiogenic and anti-inflammatory effects. MSCs can show immunosuppressive, anti- 
a)

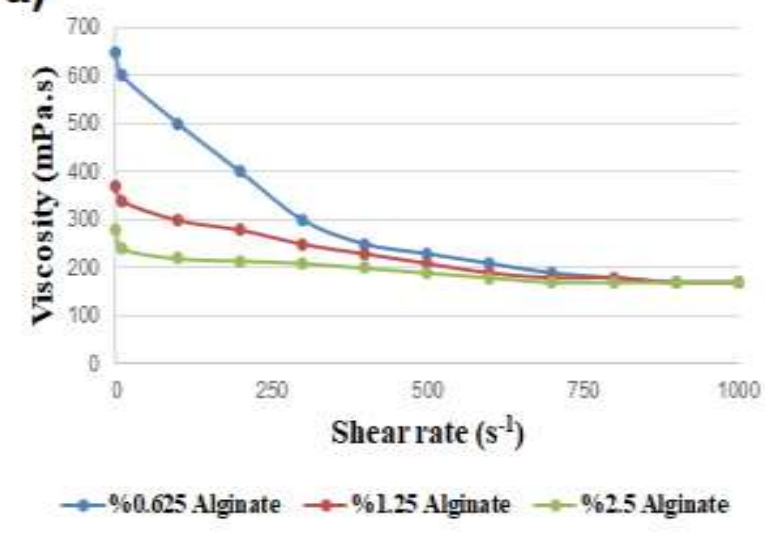

Figure 2. Alginate viscosity and deformation.

a)

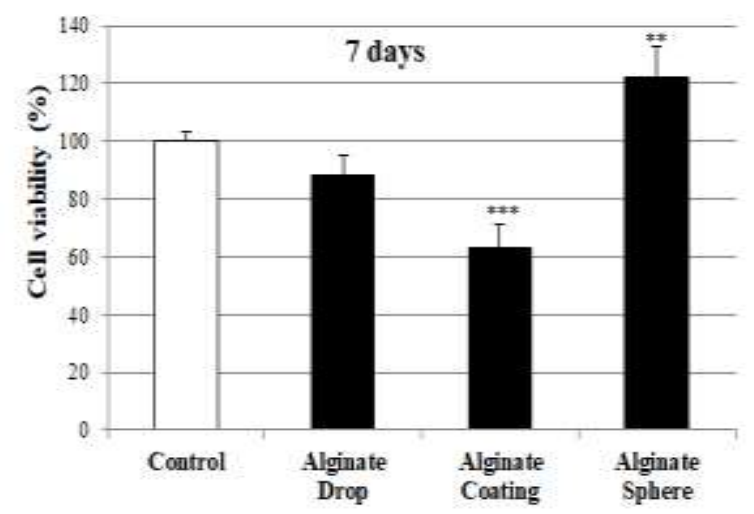

b)

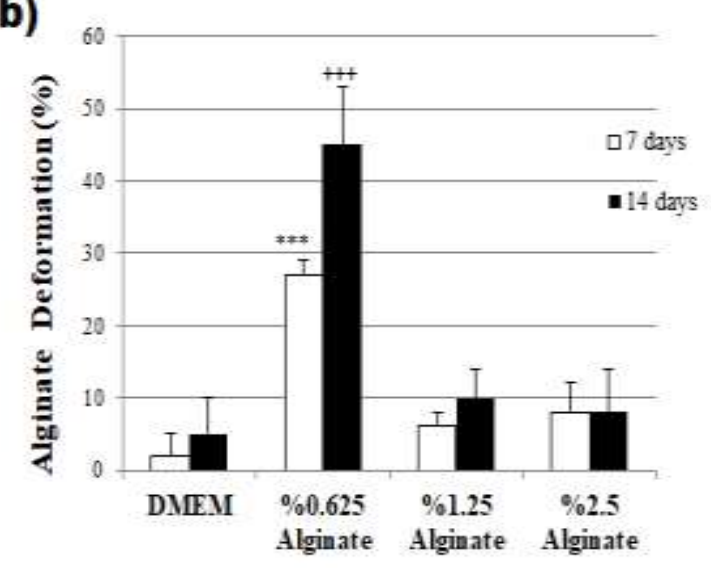

b)

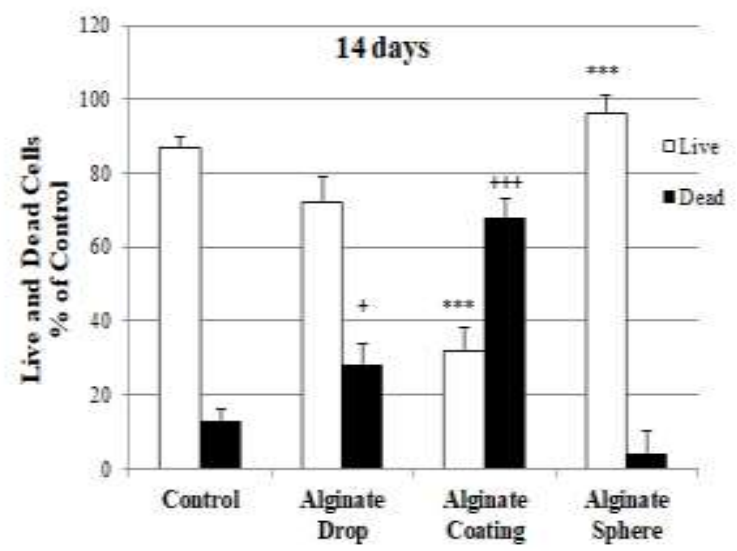

Figure 3. Effects of alginate encapsulation models in cell viability and live/dead cell ratio on UCMSC.

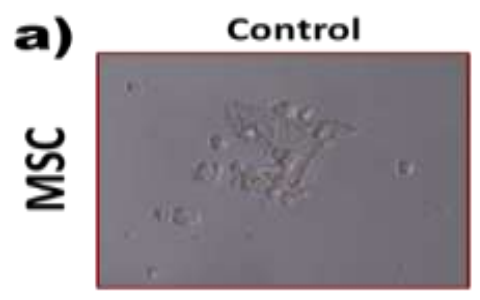

Alginate Drop
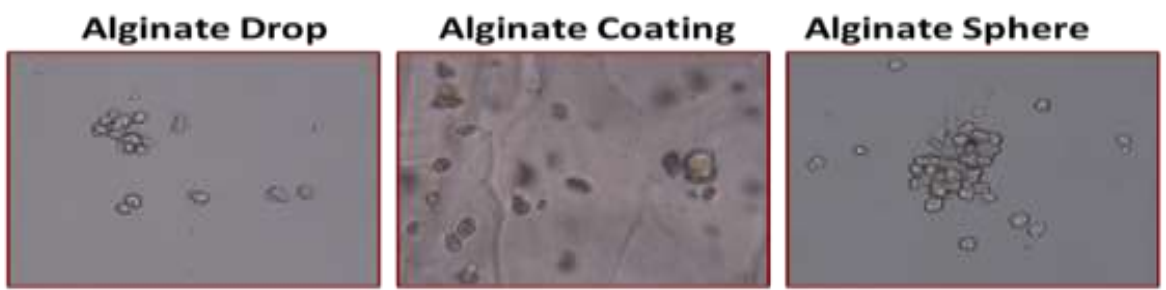

b)

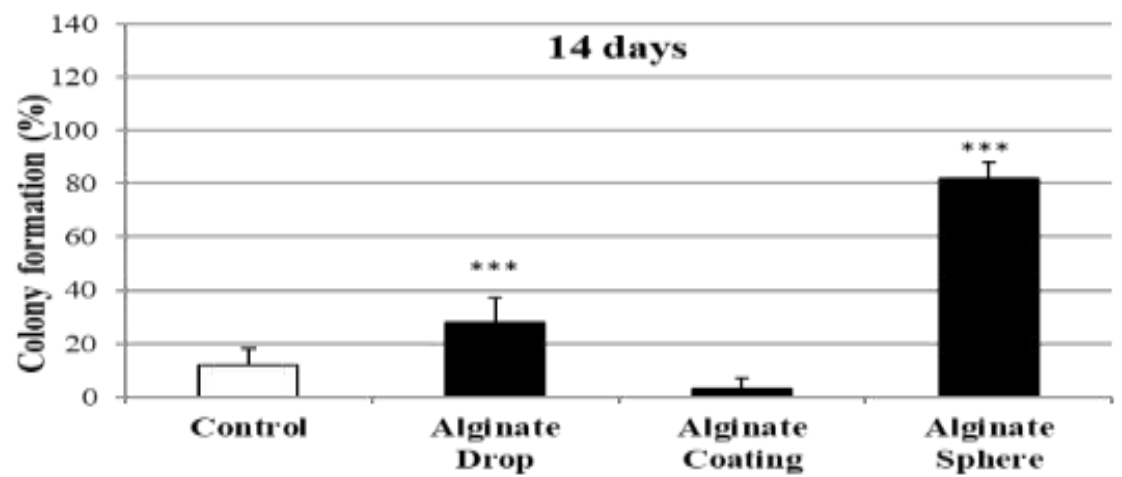

Figure 4. Effects of alginate encapsulation models in cell colony formation on UC-MSC. 
apoptotic, anti-fibrotic effects on other cells. To benefit from these potentials MSC cells, are being developed, and new therapeutic dosing studies with encapsulation are carried out in this area for cellular therapy methods $[15,16]$. For MSC cells to be used therapeutically, there is a need for an encapsulation system that can maintain their viability for a long time. Especially in MSCs, UC-derived cells that are small amount but have the most incredible growth ability and contain important biofactors that are essential. UC-MSCs are isolated from umbilical cord blood or cord tissue. MSC isolation from UC tissue requires a lengthy dissection step, and opening the umbilical cord and manual removal of the vessels before mincing Wharton jelly is time-consuming and increases the risk of contamination. UC-MSCs are essential to be isolated under GMP conditions and stored for long periods [17]. UCMSCs have significant enhancements, have high proliferation potentials, vast differentiation potentials, and enhanced immune modulation properties [18,19]. For these reasons, UC-MSCs have high therapeutic potential and have an important place in clinical trials worldwide [20,21]. Encapsulation of cells is a technique that enables living cells to be confined with unique biopolymeric materials and use them in the potential treatment of various human diseases [22]. There are many studies on low immunity for cell encapsulation and the development of biomaterials to protect stem cells [23]. It is crucial to develop a method for both the isolation of stem cells and their long-term storage. The properties and shapes of biomaterials during production are essential for clinical applications of cell encapsulation of stem cells [24,25]. Many in vivo studies showed that the encapsulation and administration of stem cells produced from different sources (such as bone marrow, adipose tissue) with alginate [26]. For example, alginate-encapsulated human BM-MSCs have demonstrated a therapeutically curative effect at the infarct site in the rat myocardial infarction model [27]. Another study showed that alginate-encapsulated MSCs remained viable for 30 days and did not lose their therapeutic effect when administered subcutaneously to mice [28]. Similarly, in our study, we found that the alginate sphere group's vitality was high on the 14th day. On the other hand, some suggestions would be beneficial to use a single cell microgel encapsulation approach for MSC. In systemic applications of MSC, it provides diffusion limitations in protecting stem cells against hypoxic effects and facilitating molecules released from the cell [29]. There are still restrictive studies regarding colony formation in stem cells in single-cell capsulations [30]. Colony formation of mesenchymal stem cells (maybe called spheroidization) exhibits improved therapeutic potential in vitro, but if spheroids fail to attach in the environment, they leave control of cell function to the extracellular matrix and potentially limit development time. Using biomaterials is supported by spheroid cell transmission, cell retention and stem cells' functions [31]. In the study using the osteoarthritis model, it was reported that allogeneic rat MSC cells survived longer when encapsulated in alginate and showed metabolic activity for at least eight weeks in vivo [32]. In this study, high and low weight alginate and allogenic MSC cells were first mixed in the form of beads and marked with gadolinium for monitoring. It has a similar structure to the drop alginate complexes in our study and is formed with different cells. The production of cellalginate microcapsules containing MSC cells in a standardized, safe manner must certainly establish the "ready-to-use" potential in clinical 
practice [29]. Some researchers, MSC cells isolated from the umbilical cord Wharton jelly were encapsulated with alginate, and differentiation into neuron-like cells was examined. The study showed that alginate could effectively induce neuronal differentiation in a three-dimensional cell culture system by protecting cells [33]. In a different study, MSC cells isolated from Wharton jelly were encapsulated with alginate, and the interleukins, chemokines, growth factors, and soluble forms of adhesion molecules released into the environment were investigated. It has been reported that alginate does not change cells' morphological properties and can provide protein circulation in the general life cycle [34].

\section{Conclusion}

For bio fabrication studies, UC-MSC provides dissemination of research on encapsulation. The alginate sphere in our study was created with the logic of microencapsulation. It has been determined that the cells placed in the capsules can form a better colony. When the biodegradable alginate system is opened in the tissues, these colonies' potential to form new cells there will be higher. This method may be more useful for biofabrication studies. For smaller microencapsulation studies, these data will shed light on future studies.

\section{Acknowledgments:}

This study has been supported by a grant (120S682) from the Scientific and Technological Research Council of Turkey (TUBITAK) and Adnan Menderes University Research Grant (TPF-20021) to Ozge Cevik. We are grateful to ODC Research and Development Inc for providing the UC-MSC cell lines.
Conflict of Interest: The authors declare that they have no conflict of interest.

Ethical statement: Since it is a cell culture study and commercial line is used, ethics committee permission is not required.

\section{Open Access Statement}

This is an open access journal which means that all content is freely available without charge to the user or his/her institution under the terms of the Creative Commons Attribution NonCommercial License (http://creativecommons.org/licenses/bync/4.0). Users are allowed to read, download, copy, distribute, print, search, or link to the full texts of the articles, without asking prior permission from the publisher or the author.

\section{References}

[1]Le Blanc K, Mougiakakos D. Multipotent mesenchymal stromal cells and the innate immune system. Nat Rev Immunol. 2012;12(5):383-96.

[2]Diekman B, Wu C-L, Louer C, et al. Intraarticular Delivery of Purified Mesenchymal Stem Cells From C57BL/6 or MRL/MpJ Superhealer Mice Prevents Posttraumatic Arthritis. Cell Transplant. 2013; 22(8):1395408.

[3]Pers Y-M, Rackwitz L, Ferreira R, et al. Adipose Mesenchymal Stromal Cell-Based Therapy for Severe Osteoarthritis of the Knee: A Phase I Dose-Escalation Trial. Stem Cells Transl Med. 2016; 5(7):847-56.

[4]Harrell C, Fellabaum C, Jovicic N, et al. Molecular Mechanisms Responsible for Therapeutic Potential of Mesenchymal Stem Cell-Derived Secretome. Cells. 201;8(5):467.

[5]Wagner B, Henschler R. Fate of Intravenously Injected Mesenchymal Stem Cells and Significance for Clinical 
Application. Adv Biochem Eng Biotechnol. 2013; 130:19-37.

[6]Hmadcha A, Martin-Montalvo A, Gauthier BR, et al. Therapeutic Potential of Mesenchymal Stem Cells for Cancer Therapy. Front Bioeng Biotechnol. 2020;8:43.

[7]Van Pham P. Stem cell drugs: the next generation of pharmaceutical products. Biomed Res Ther. 2016; 3(10):857-71.

[8]Selich A, Zimmermann K, Tenspolde M, et al. Umbilical cord as a long-Term source of activatable mesenchymal stromal cells for immunomodulation. Stem Cell Res Ther. 2019; 10(1):285.

[9]Chen M-Y, Lie P-C, Li Z-L, et al. Endothelial differentiation of Wharton's jelly-derived mesenchymal stem cells in comparison with bone marrow-derived mesenchymal stem cells. Exp Hematol. 2009; 37(5):629-40.

[10]Hendijani F, Sadeghi-Aliabadi H, Javanmard S. Comparison of human mesenchymal stem cells isolated by explant culture method from entire umbilical cord and Wharton's jelly matrix. Cell Tissue Bank. 2014; 5(4):555-65.

[11]Leijs MJ, Villafuertes E, Haeck JC, et al. Encapsulation of allogeneic mesenchymal stem cells in alginate extends local presence and therapeutic function. Eur Cell Mater. 2017; 33:43-58.

[12]Hunt NC, Smith AM, Gbureck U, et al. Encapsulation of fibroblasts causes accelerated alginate hydrogel degradation. Acta Biomater. 2010; 6(9):3649-56.

[13] Orive G, Hernández RM, Gascón AR, et al. History, Challenges and Perspectives of Cell Microencapsulation. Trends Biotechnol. 2004;22(2):87-92.
[14]Cevik O, Turut FA, Acidereli H, et al. Cyclosporine-A induces apoptosis in human prostate cancer cells PC3 and DU145 via downregulation of COX-2 and upregulation of TGF $\beta$. Turkish J Biochem. 2019; 44(1):47-54.

[15] Murray IR, Chahla J, Safran MR, et al. International Expert Consensus on a Cell Therapy Communication Tool: DOSES. J Bone Jt Surg. 2019;101(10):904-11.

[16] Pittenger MF, Discher DE, Péault BM, et al. Mesenchymal stem cell perspective: cell biology to clinical progress. NPJ Regen Med. 2019;4:22.

[17] Smith JR, Pfeifer K, Petry F, et al. Standardizing Umbilical Cord Mesenchymal Stromal Cells for Translation to Clinical Use: Selection of GMPCompliant Medium and a Simplified Isolation Method. Stem Cells Int. 2016; 2016:6810980.

[18]Zeddou M, Briquet A, Relic B, et al. The umbilical cord matrix is a better source of mesenchymal stem cells (MSC) than the umbilical cord blood. Cell Biol Int. 2010; 34(7):693-701.

[19]Bárcia R, Santos J, Filipe M, et al. What Makes Umbilical Cord Tissue-Derived Mesenchymal Stromal Cells Superior Immunomodulators When Compared to Bone Marrow Derived Mesenchymal Stromal Cells? Stem Cells Int. 2015; 2015:583984.

[20] Sharma RR, Pollock K, Hubel A, et al. Mesenchymal stem or stromal cells: a review of clinical applications and manufacturing practices. Transfusion. 2014; 54(5):1418-37.

[21]Meng F, Xu R, Wang S, et al. Human umbilical cord-derived mesenchymal stem cell therapy in patients with COVID-19: a 
phase 1 clinical trial. Signal Transduct Target Ther. 2020; 5(1):172.

[22] Waheed A, Mazumder MAJ, Al-Ahmed A, et al. Cell Encapsulation BT - Functional Biopolymers. In: Jafar Mazumder MA, Sheardown H, Al-Ahmed A, editors. Cham: Springer International Publishing; 2019. p. 377-427.

[23] Li H, Koenig AM, Sloan P, et al. In vivo assessment of guided neural stem cell differentiation in growth factor immobilized chitosan-based hydrogel scaffolds. Biomaterials. 2014; 35(33):9049-57.

[24] Sart S, Ma T, Li Y. Preconditioning stem cells for in vivo delivery. Biores Open Access. 2014; 3(4):137-49.

[25] Weick JP. Functional Properties of Human Stem Cell-Derived Neurons in Health and Disease. Stem Cells Int. 2016; 2016:4190438.

[26] Kim I, Lee SK, Yoon JI, et al. Fibrin glue improves the therapeutic effect of MSCs by sustaining survival and paracrine function. Tissue Eng Part A. 2013; 19(21-22):237381.

[27]Levit RD, Landázuri N, Phelps EA, et al. Cellular encapsulation enhances cardiac repair. J Am Heart Assoc. 2013;2(5):e000367.

[28]Zanotti L, Sarukhan A, Dander E, et al. Encapsulated mesenchymal stem cells for in vivo immunomodulation. Leukemia. 2013; 27(2):500-3.

[29] Mao AS, Özkale B, Shah NJ, et al. Programmable microencapsulation for enhanced mesenchymal stem cell persistence and immunomodulation. Proc Natl Acad Sci U S A. 2019; 116(31):1539297.

[30] Kamperman T, Karperien M, Le Gac S, et al. Single-Cell Microgels: Technology,
Challenges, and Applications. Trends Biotechnol. 2018; 36(8):850-65.

[31] Ho SS, Murphy KC, Binder BYK, et al. Increased Survival and Function of Mesenchymal Stem Cell Spheroids Entrapped in Instructive Alginate Hydrogels. Stem Cells Transl Med. 2016; 5(6):773-81.

[32] Khatab S, Leijs MJ, van Buul G, et al. MSC encapsulation in alginate microcapsules prolongs survival after intra-articular injection, a longitudinal in vivo cell and bead integrity tracking study. Cell Biol Toxicol. 2020; 36(6):553-70.

[33] Hosseini SM, Vasaghi A, Nakhlparvar N, et al. Differentiation of Wharton's jelly mesenchymal stem cells into neurons in alginate scaffold. Neural Regen Res. 2015; 10(8):1312-16.

[34]Penolazzi L, Tavanti E, Vecchiatini R, et al. Encapsulation of Mesenchymal Stem Cells from Wharton's Jelly in Alginate Microbeads. Tissue Eng Part C Methods. 2009; 16(1):141-55. 